\title{
Effect of AMP-activated protein kinase activation on cardiac fibroblast proliferation induced by coxsackievirus B3
}

\author{
SHENGYANG JIANG $^{1}$, SHUNLI TIAN ${ }^{2}$, XUEMING WU $^{1}$, YIJIA TAO ${ }^{1}$ and DONGLIN JIANG ${ }^{3}$ \\ ${ }^{1}$ Department of Cardiology, The Third Affiliated Hospital of Nantong University, \\ Wuxi Institute of Integrated Traditional Chinese and Western Medicine, Wuxi, Jiangsu 214041; \\ ${ }^{2}$ Department of Geratology, Tianjin Geriatric Institute, Tianjin Medical University General Hospital, Tianjin 300052; \\ ${ }^{3}$ Clinical Central Laboratory, The Third Affiliated Hospital of Nantong University, \\ Wuxi Institute of Integrated Traditional Chinese and Western Medicine, Wuxi, Jiangsu 214041, P.R. China
}

Received January 28, 2015; Accepted March 1, 2016

DOI: $10.3892 /$ etm.2016.3174

\begin{abstract}
Excessive fibroblast proliferation and collagen production are the major pathogenic mechanisms in the progression of viral myocarditis, which is most frequently associated with infection by coxsackievirus B3 (CVB3). AMP-activated protein kinase (AMPK) has been confirmed to be involved in the progression of myocardial remodeling. However, it remains unclear whether AMPK has an effect on CVB3-induced cardiac fibroblast proliferation. In the present study, the effects of AMPK on cardiac fibroblast proliferation and collagen secretion induced by CVB3 were investigated. Proliferation of neonatal cardiac fibroblasts was determined by cell counting and detection of newly synthesized DNA in cells, and the proportion of cells in the $\mathrm{S}$ phase was measured. Hydroxyproline ELISA was used to detect collagen secretion. Phosphorylation of AMPK $\alpha-\mathrm{Th}^{172}$ was evaluated by western blotting. It was found that neonatal cardiac fibroblasts were clearly proliferating markedly and secreting collagen at $24 \mathrm{~h}$ after CVB3 infection, and peaked at $48 \mathrm{~h}$. These effects were inhibited following pretreatment of the fibroblasts with 5-aminoimidazole-4-carboxamide-ribonucleoside (AICAR), a specific AMPK activator, for $2 \mathrm{~h}$ prior to CVB3 infection. However, if the cells were preincubated with compound $\mathrm{C}$ for $30 \mathrm{~min}$, the inhibitive effects of AICAR were reversed. Western blotting results indicated that AMPK $\alpha$-Thr ${ }^{172}$ phosphorylation was increased by AICAR and attenuated by Compound $\mathrm{C}$. The results of the present study suggest that CVB3 infection increases cardiac fibroblast proliferation and collagen secretion, and that these phenomena can be
\end{abstract}

Correspondence to: Professor Donglin Jiang, Clinical Central Laboratory, The Third Affiliated Hospital of Nantong University, Wuxi Institute of Integrated Traditional Chinese and Western Medicine, 585 Xing-Yuan North Road, Beitang, Wuxi, Jiangsu 214041, P.R. China

E-mail: jsystar@126.com

Key words: AMP-activated protein kinase, coxsackievirus, cardiac fibroblast, collagen, cell proliferation inhibited by activated AMPK. These findings contribute to our understanding of AMPK function and the future design of therapeutic approaches for the treatment of cardiac fibrosis caused by chronic viral infection, such as CVB3-induced myocarditis.

\section{Introduction}

Viral myocarditis is a potentially lethal infection often resulting in arrhythmia, heart failure, dilated cardiomyopathy and mortality (1). There are three phases of viral myocarditis. During the early phase, viral infection and replication cause direct damage to the myocardial cells. Autoimmune myocarditis, which is characterized by autoimmune injury induced by the viral infection, is the second stage and involves the expression and secretion of numerous inflammatory cytokines and chemokines. Finally, in the last phase, inflammation progressively subsides, although the viral genomes persist in the heart (2). Myocardial fibrosis and pathological remodeling, which lead to impaired heart function, may occur during the early and late stages of viral myocarditis. Coxsackievirus of group B3 (CVB3) is one of the most common cardiotropic viruses and is among numerous known etiologies for myocarditis $(3,4)$. In the pathology of CVB3-induced myocarditis, cardiac fibroblasts are an important contributor to virus replication-mediated aggravation of myocarditis (5); they are central mediators of inflammatory and fibrotic myocardial remodeling in the injured and failing heart (6).

Adenosine monophosphate-activated protein kinase (AMPK) is a key regulator of cellular energy homeostasis, and is involved in multiple anabolic and catabolic signaling pathways in myocardial cells (7). It has been reported that the long-term activation of AMPK was able to attenuate angiotensin II-induced or pressure-overload cardiac hypertrophy $(8,9)$. In addition, the association between AMPK and virus infection has been investigated, and it was demonstrated that activation of AMPK was able to restrict CVB3 replication by inhibiting lipid accumulation (10). However, it remains unclear whether AMPK has an effect on CVB3-induced inflammatory cardiomyopathy and myocardial fibrosis. Therefore, the present study aimed to investigate the effect of 
AMPK activation on cardiac fibroblast proliferation following CVB3 infection.

\section{Materials and methods}

Reagents. 5-Aminoimidazole-4-carboxamide-ribonucleoside (AICAR) was purchased from Toronto Research Chemicals (Toronto, ON, Canada). Compound $\mathrm{C}$ was purchased from EMD Millipore (San Diego, CA, USA). Antibodies against phospho-AMPK $\alpha$-Thr ${ }^{172}$ (p-AMPK $\alpha-$ Thr $^{172}$ ) were from Cell Signaling Technology, Inc. (Beverly, MA, USA). Antibodies against glyceraldehyde 3-phosphate dehydrogenase (GAPDH) were from Santa Cruz Biotechnology, Inc. (Dallas, TX, USA).

Determination of virus titers. The CVB3 Nancy strain (cat. no. VR30; ATCC, Manassas, VA, USA) was propagated four times in HeLa cells (cat. no. CCL-2; ATCC). The infected HeLa cells were broken up by three cycles of freezing and thawing. Then, debris was removed, and serial dilutions (10-fold) of the supernatants were prepared in infection medium, which comprised minimal essential medium (GE Healthcare Life Sciences, Logan, UT, USA) for HeLa cells or fibroblasts, $2 \%$ fetal bovine serum (FBS; GE Healthcare Life Sciences), $30 \mathrm{mM}$ $\mathrm{MgCl}_{2}, 100 \mathrm{U} / \mathrm{ml}$ penicillin, $100 \mu \mathrm{g} / \mathrm{ml}$ streptomycin sulfate (both Gen-View Scientific, Inc., El Monte, CA, USA) and $2 \mathrm{mM}$ glutamine (Amresco LLC, Solon, OH, USA). Subsequently, the samples were transferred onto subconfluent monolayers of HeLa cells grown in 96-well culture plates containing $100 \mathrm{ml}$ infection medium. Following incubation at $37^{\circ} \mathrm{C}$ for $24 \mathrm{~h}$, cells were stained with $0.5 \%$ crystal violet (in water) for $5 \mathrm{~min}$. The $50 \%$ tissue culture infectious dose (TCID50) was calculated. The tissue culture infectious dose infecting $50 \%$ of the cells was calculated using the Reed-Muench method (11).

Cell isolation and culture. Cardiac fibroblasts were isolated from neonatal Sprague Dawley rats (age, 1 day). Minced ventricles were digested with $0.1 \%$ trypsin and $0.03 \%$ collagenase II, and the cells were collected and placed in $10-\mathrm{cm}$ cell culture plates containing $10 \mathrm{ml}$ Dulbecco's modified Eagle's medium (DMEM; GE Healthcare Life Sciences) supplemented with $1 \%$ penicillin-streptomycin and $10 \%$ FBS. After $60 \mathrm{~min}$ in a $37^{\circ} \mathrm{C}$ incubator, cardiac fibroblasts attached to the culture plates. Then, the fibroblasts were washed twice and cultured in DMEM with $10 \% \mathrm{FBS}$ at $37^{\circ} \mathrm{C}$ for $48 \mathrm{~h}$ until they reached confluence. Subsequently, cultured cells were confirmed to be pure cardiac fibroblasts by morphological inspection. All the cardiac fibroblasts were incubated in DMEM with $2 \%$ FBS for $24 \mathrm{~h}$ before the experiments were performed. The experiments were as follows: i) To explore the effects of CVB3 on cardiac fibroblasts, neonatal cardiac fibroblasts $\left(5 \times 10^{4}\right.$ cells/well) in serum-free DMEM medium were infected with CVB3 (100 TCID50) for 24, 48 and $72 \mathrm{~h}$; ii) To explore the effect of activated AMPK on cardiac fibroblast proliferation and collagen secretion, the fibroblasts were pretreated with $1 \mathrm{mmol} / 1$ AICAR (a specific AMPK activator) for $2 \mathrm{~h}$, then infected with CVB3 (100 TCID50) for $48 \mathrm{~h}$; and iii) the fibroblasts were preincubated with $1 \mu \mathrm{mol} / 1$ Compound $\mathrm{C}$ (a specific AMPK inhibitor) for $30 \mathrm{~min}$, then treated with AICAR (1 mmol/l) and CVB3 (100 TICD50) in succession. The animal care and experimental protocols were in compliance with the
Animal Management Rule of the People's Republic of China (Ministry of Health, China; document no. 552; http://www.gov. cn/gongbao/content/2011/content_1860757.htm) and the study was approved by the Animal Care Committee of the Third Affiliated Hospital of Nantong University (Wuxi, China).

Cell proliferation assay. Cell counting and detection of 5-bromo-2'-deoxyuridine (BrdU) incorporation were used to assess cellular proliferation. Briefly, cells were detached using $0.25 \%$ trypsin (Invitrogen; Thermo Fisher Scientific, Inc., Waltham, MA, USA). Following centrifugation at 3,000 $\mathrm{x}$ for $3 \mathrm{~min}$ at $4^{\circ} \mathrm{C}$, the cells were resuspended in phosphate-buffered saline (PBS), after which $25 \mu \mathrm{l}$ aliquots of the cell suspension were mixed with an equal volume of $0.4 \%$ trypan blue dye (Sigma-Aldrich, St. Louis, MO, USA) at room temperature for 3 min. Finally, the samples were placed in a hemocytometer (Neubauer improved cell counting chamber; Paul Marienfeld GmbH \& Co. KG; Lauda-Königshofen, Germany) and counted under an Olympus IX73 microscope (Olympus Corporation, Tokyo, Japan). The BrdU incorporation assay was performed using a colorimetric BrdU cell proliferation enzyme-linked immunosorbent assay (ELISA) kit for rats (Roche, Mannheim, Germany) according to the manufacturer's protocol.

Flow cytometric assessment of cell cycle. The cell samples were washed twice with cold PBS, then fixed with $70 \%$ ethanol. After allowing to stand at $4^{\circ} \mathrm{C}$ overnight, the samples were resuspended with PBS including $0.1 \mathrm{mg} / \mathrm{ml}$ RNase (Sigma-Aldrich). Subsequently, cells were incubated at $37^{\circ} \mathrm{C}$ for $30 \mathrm{~min}$. After that, $50 \mathrm{mg} / \mathrm{ml}$ propidium iodide was used to suspend the cells. Finally, the cell samples were analyzed by flow cytometry (FACSCalibur ${ }^{\mathrm{TM}}$; BD Biosciences, San Jose, CA, USA), and the cell cycle distribution, specifically the percentage of cells in the $\mathrm{S}$ phase, was determined using ModFit LT cell cycle analysis software (Verity Software House, Topsham, ME, USA).

Hydroxyproline measurement. A rat hydroxyproline ELISA kit (Kaibo Biochemical Reagents Co., Ltd., Shanghai, China) was used to detect collagen levels on the basis of the content of its major component, hydroxyproline. Hydroxyproline concentration was calculated according to the OD value at $450 \mathrm{~nm}$ provided using an ELISA plate reader (Model 550; Bio-Rad Laboratories, Inc., Hercules, CA, USA).

Western blot analysis. Cell samples were washed once with cold PBS following the treatment. Subsequently, buffer [100 mmol/1 NaCl, $10 \mathrm{mmol} / 1$ sodium pyrophosphate, $1 \mathrm{mmol} / 1$ sodium vanadate, $50 \mathrm{mmol} / \mathrm{l} \mathrm{NaF}, 5 \mathrm{mmol} / \mathrm{l}$ ethylenediamine tetra-acetic acid, $1 \%$ sodium deoxycholate, $20 \mathrm{mmol} / 1 \mathrm{Tris}-\mathrm{HCl}$ (pH 7.4), 0.1\% sodium dodecyl sulfate (SDS), $1 \mathrm{mmol} / 1$ phenylmethylsulfonyl fluoride, $0.1 \mathrm{mmol} / \mathrm{l}$ aprotinin, $1 \mathrm{mmol} / \mathrm{l}$ leupeptin, $1 \%$ Triton $\mathrm{X}-100$ and $10 \%$ glycerol) was used to lyse the cell samples. The protein concentration was estimated using a BCA protein assay kit (Pierce Biotechnology, Rockford, IL, USA). Protein samples (30 mg) were loaded and separated on a $10 \%$ SDS-polyacrylamide gel and then transferred electrophoretically to nitrocellulose membranes (Pall Corporation, East Hill, NY, USA). Nonspecific binding sites were blocked using 5\% (w/v) non-fat dry milk in Tris-buffered 
A

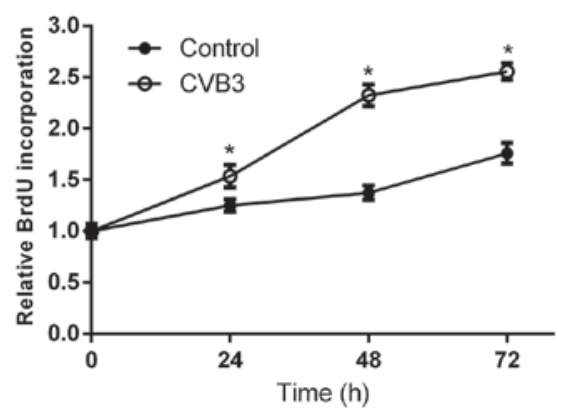

B

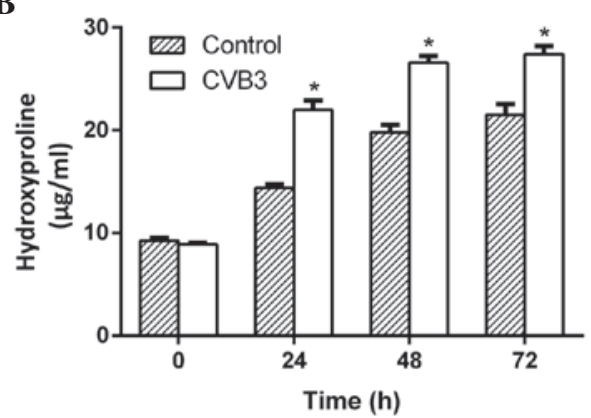

Figure 1. Cell proliferation and hydroxyproline secretion were promoted by coxsackievirus B3 (CVB3) in neonatal rat cardiac fibroblasts. The fibroblasts were infected with CVB3 for 24, 48 and $72 \mathrm{~h}$, and then (A) 5-bromo-2'-deoxyuridine (BrdU) incorporation and (B) hydroxyproline secretion were assessed. Data are presented as the mean \pm standard error of the mean $(n=3)$. ${ }^{*} \mathrm{P}<0.05$ vs. the control group at the same time point.

A

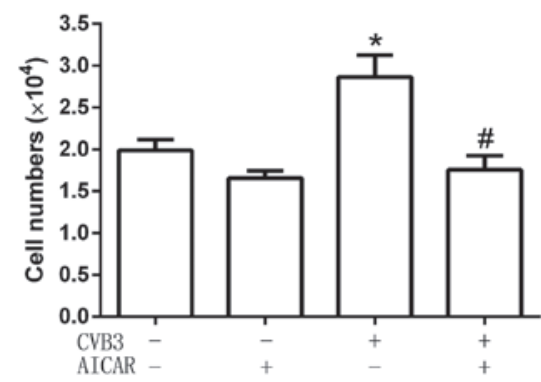

C

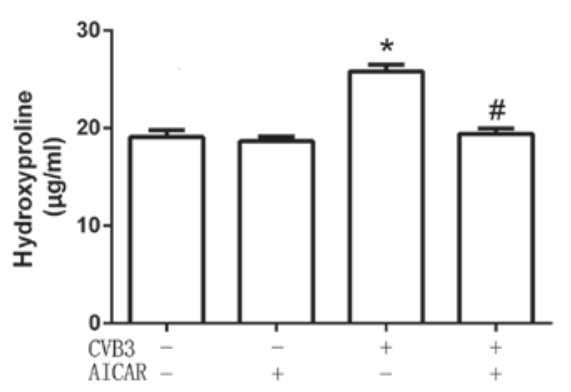

B

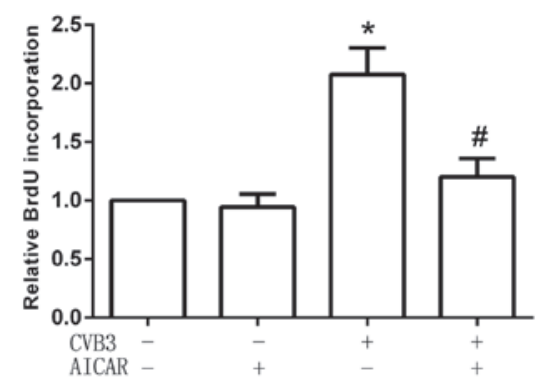

D

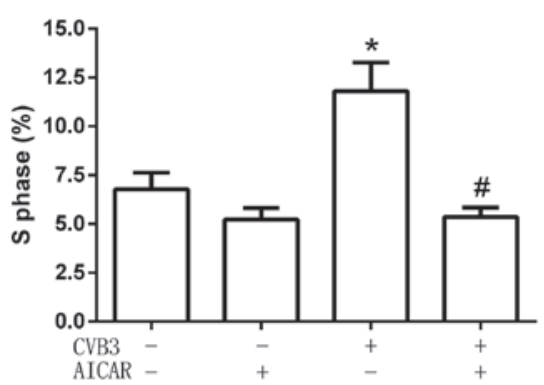

Figure 2. AICAR inhibited cell proliferation and hydroxyproline secretion promoted by coxsackievirus B3 (CVB3) in neonatal rat cardiac fibroblasts. After fibroblasts were pretreated with AICAR and infected with CVB3 for $48 \mathrm{~h}$ (A) cell numbers were counted, and (B) 5-bromo-2'-deoxyuridine (BrdU) incorporation and (C) hydroxyproline secretion were assessed. (D) In addition, cells were fixed, stained with propidium iodide, and analyzed for DNA content by flow cytometry (data were obtained from 15,000 events and represented as percentage of cells in the $\mathrm{S}$ phase). Data are the mean \pm standard error of the mean ( $\mathrm{n}=3$ ). ${ }^{*} \mathrm{P}<0.05$ vs. the control group; ${ }^{*} \mathrm{P}<0.05$ vs. the CVB3 group. AICAR, 5-aminoimidazole-4-carboxamide-ribonucleoside.

saline with $0.1 \%$ Tween-20 (TBST) buffer solution $(0.138 \mathrm{~mol} / 1$ $\mathrm{NaCl}, 20 \mathrm{mmol} / \mathrm{l}$ Tris-HCl, $\mathrm{pH} 7.6,0.1 \%$ [v/v] Tween-20; Gen-View Scientific, Inc.) at room temperature for $1 \mathrm{~h}$. Then, the membranes were incubated overnight at $4{ }^{\circ} \mathrm{C}$ with rabbit anti-p-AMPK $\alpha$-Thr172 (1:1,000; cat. no. 2531) and mouse anti-GAPDH (1:3,000; cat. no. sc-32233) monoclonal antibodies. After washing three times with TBST, the membranes were incubated with horseradish peroxidase-conjugated goat anti-rabbit IgG (1:6,000; cat. no. sc-2004; Santa Cruz Biotechnology, Inc.) and goat anti-mouse $\operatorname{IgG}(1: 6,000$; cat. no. sc-2005; Santa Cruz Biotechnology, Inc.) for $1 \mathrm{~h}$ at room temperature. An enhanced chemiluminescence detection kit (cat. no. 32106; Pierce Biotechnology, Inc., Rockford, IL, USA) was used to visualize the immunoreactive bands on the membranes, according to the manufacturer's protocol. The chemiluminescence signal was detected by exposure to X-ray film (Kodak, Rochester, NY, USA). Densitometry analysis was used to quantify the protein bands by calculating the band density using Scion Image software, version 4.03 (Scion Corporation, Frederick, MD, USA). All densitometry data are expressed as fold-change from the control.

Statistical analysis. Values are expressed as the mean \pm standard error of the mean. The statistical significance of differences was determined using one-way analysis of variance for multiple comparisons with Tukey's post hoc test for analysis between groups. $\mathrm{P}<0.05$ was considered significant.

\section{Results}

CVB3 promotes cardiac fibroblast proliferation and collagen (hydroxyproline) secretion. To determine whether CVB3 promoted cardiac fibroblast proliferation and collagen secretion, neonatal rat cardiac fibroblasts incubated in serum-free DMEM medium were infected with CVB3 (100 TCID50) for 24, 48 and 72 h. Then, newly synthesized DNA in replicating 
A

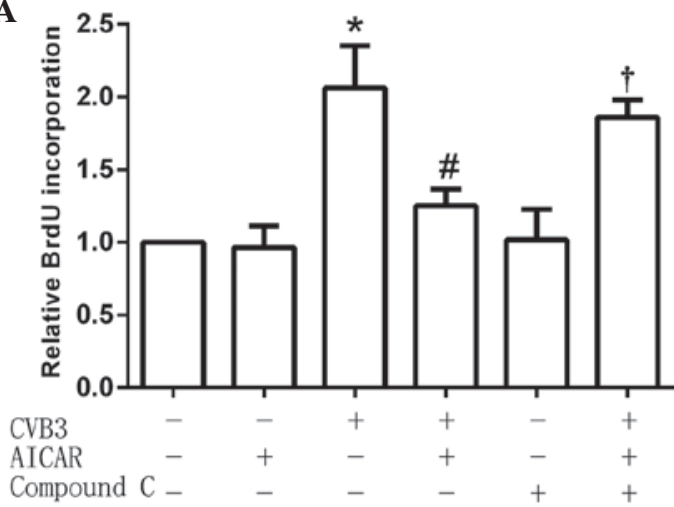

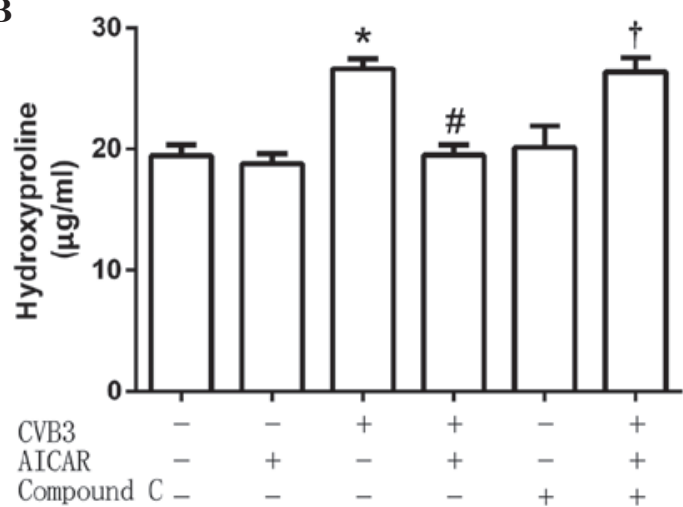

Figure 3. Compound $\mathrm{C}$ inhibited the effects of AICAR in the neonatal rat cardiac fibroblasts infected by coxsackievirus B3 (CVB3). Following incubation with compound $\mathrm{C}(10 \mu \mathrm{M})$ for $1 \mathrm{~h}$, fibroblasts were treated with AICAR and then infected with CVB3 for $48 \mathrm{~h}$. (A) BrdU incorporation and (B) hydroxyproline secretion were assessed. Data are the mean \pm standard error of the mean $(n=3)$. ${ }^{*} \mathrm{P}<0.05$ vs.the control group; ${ }^{\#} \mathrm{P}<0.05$ vs. the $\mathrm{CVB} 3$ group; ${ }^{\dagger}<0.05$ vs. the CVB3 + AICAR group. AICAR, 5-aminoimidazole-4-carboxamide-ribonucleoside.
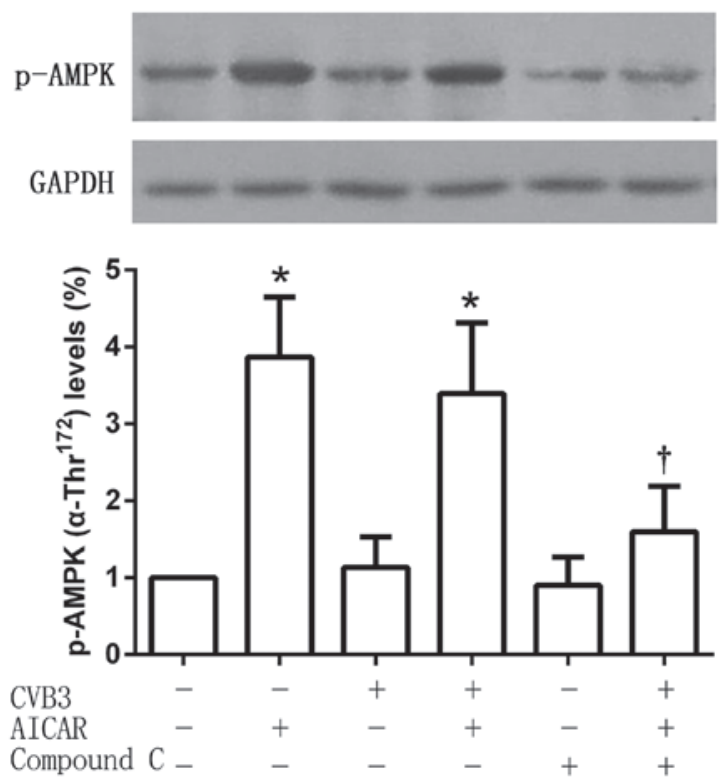

Figure 4. Differential phosphorylation levels of AMP-activated protein kinase (AMPK) $\alpha-$ Thr $^{172}$ in all the neonatal rat cardiac fibroblast groups. Cells were incubated with compound $\mathrm{C}(10 \mu \mathrm{M})$ for $1 \mathrm{~h}$ in advance, then treated with AICAR and infected with CVB3 for $48 \mathrm{~h}$. Phosphorylation levels of AMPK $\alpha-$ Thr $^{172}$ were detected, and the same membrane was stripped and reprobed with GAPDH antibody. Data are given as the mean \pm standard error of the mean $(n=3) .{ }^{*} \mathrm{P}<0.05$ vs. the control group; ${ }^{\dagger} \mathrm{P}<0.05$ vs. the CVB3 + AICAR group. AICAR, 5-aminoimidazole-4-carboxamide-ribonucleoside; CVB3, coxsackievirus B3; GAPDH, glyceraldehyde-3-phosphate dehydrogenase.

cells and hydroxyproline content in the culture supernatant were measured. After $24 \mathrm{~h}$ of infection, both the newly synthesized DNA (Fig. 1A) and hydroxyproline (Fig. 1B) increased significantly in the samples, and reached a peak at $48 \mathrm{~h}$ after infection. This phenomenon indicated that CVB3 induced cardiac fibroblast proliferation and collagen secretion.

AICAR inhibits cardiac fibroblast proliferation and collagen secretion induced by $C V B 3$. To explore the effect of activated AMPK on cardiac fibroblast proliferation and collagen secretion, cells were pretreated with the specific AMPK activator AICAR (1 mmol/l) for $2 \mathrm{~h}$, then infected with
CVB3 (100 TCID50) for $48 \mathrm{~h}$. Cell numbers, DNA synthesis, cell cycles and hydroxyproline content were detected. The results showed that, following pretreatment with AICAR, CVB3 did not increase cell numbers (Fig. 2A), the quantity of newly synthesized DNA in cells (Fig. 2B), hydroxyproline content in the supernatant (Fig. 2C) and the proportion of cells in the $\mathrm{S}$ phase $(9.54 \pm 1.22$ for cells treated with AICAR alone vs. $5.65 \pm 0.95 \%$ for AICAR-pretreated CVB3-infected cells; Fig. 2D). These data showed that AICAR inhibited CVB3-induced proliferation in cardiac fibroblasts.

Inhibitive effects of AICAR are attenuated by compound $C$. The cardiac fibroblasts $\left(5 \times 10^{4}\right.$ per well) were preincubated with the specific AMPK inhibitor Compound C ( $1 \mu \mathrm{mol} / \mathrm{l})$ for $30 \mathrm{~min}$, then treated with AICAR $(1 \mathrm{mmol} / \mathrm{l})$ followed by CVB3 (100 TICD50). In treated cells, the activity of CVB3 was restored to increase the quantity of newly synthesized DNA in cells (Fig. 3A) and hydroxyproline content in the cell culture supernatant (Fig. 3B). These results suggest that the inhibitive effects of AICAR were attenuated by pretreatment with Compound $\mathrm{C}$.

Phosphorylation of AMPKa-Thr172 is increased by AICAR. Phospho-AMPK $\alpha-\mathrm{Thr}^{172}$ (which serves as an indicator of AMPK activity) protein expression levels were detected after treatment. As an AMPK activator, AICAR significantly increased the phosphorylation of AMPK $\alpha-\mathrm{Thr}^{172}$, an effect that was reversed by Compound $\mathrm{C}$. No significant changes of AMPK $\alpha-\mathrm{Thr}^{172}$ phosphorylation were observed in the CVB3-infected cell group compared with the untreated control, or between the CVB3 and AICAR-treated group and the AICAR-treated group (Fig. 4).

\section{Discussion}

CVB3 is the most common causative agent of myocarditis (12). In the later stage of myocarditis, persistent viral infection with CVB3 can induce the accumulation of connective tissue and extracellular matrix, chronic fibroblast activation and myocardial fibrosis $(13,14)$. CVB3 is considered to be one of the most common causes of dilated cardiomyopathy (15). 
Cardiac fibroblasts, which synthesize extracellular matrix and collagen, play a critical role in cardiac inflammation and remodeling. Compared to cardiomyocytes, cardiac fibroblasts aggravate viral myocarditis induced by CVB3 because of a higher virus replication (5). They are involved in the pathology of CVB3-induced myocarditis and dilated cardiomyopathy (5). As a major component of the protein collagen, hydroxyproline has an essential role in stabilizing the triple-helical structure of collagen, due to its effect of maximizing interchain hydrogen bond formation (16). The measurement of hydroxyproline levels can be used as an indicator of collagen content (17). In the present study, cell numbers, DNA synthesis, cell cycles and hydroxyproline content were investigated to identify the changes occurring in cardiac fibroblasts following CVB3 infection. The results confirmed that CVB3 infection promoted cardiac fibroblast proliferation and collagen secretion. This finding may contribute to our understanding of the progression from viral myocarditis to dilated cardiomyopathy.

AMPK is a heterotrimeric complex consisting of a catalytic $\alpha$-subunit and two regulatory subunits, namely $\beta$ and $\gamma$. The $\alpha$-subunit containing a serine-threonine kinase domain has a critical activating residue within the catalytic cleft $\left(\right.$ Thr $\left.^{172}\right)(18,19)$. Phosphorylation of this amino acid is essential for AMPK activity, and its phosphorylation status often is used as an indicator of the activation state of the kinase (20). As a sensor of energy status, AMPK can switch on catabolic pathways and switch off ATP-consuming processes in order to maintain cellular energy homeostasis when activated by metabolic stress (7). Recent studies have elucidated that activation of the intrinsic AMPK pathway plays an important role in the myocardial response to ischemia $(20,21)$, pressure overload (22) and heart failure (23). Although the mechanisms remain poorly understood, the pleiotropic effects of AMPK in the heart are essential to the cardioprotective actions. The compound A769662, which enhances ischemic AMPK activation, has been found to reduce infarct size in diabetic rat hearts $(24,25)$. Treatment with the AMPK activator AICAR has been demonstrated to decrease left ventricular hypertrophy induced by aortic banding in rats, although the treatment with AICAR also caused a reduction in blood pressure (26). Furthermore, metformin has been confirmed to improve the survival of patients with heart failure and type 2 diabetes by pharmacological activation of AMPK (27). Although many experiments have demonstrated that AMPK activation has pleiotropic effects in the heart, it remains unknown whether AMPK activation is involved in the viral myocarditis induced by CVB3.

In the present study, cell numbers, newly synthesized DNA and the proportion of cells in the $\mathrm{S}$ phase were significantly inhibited in the cardiac fibroblasts infected with CVB3 when the cells were pretreated with AICAR. Hydroxyproline content in the supernatant decreased, and the phosphorylation of AMPK $\alpha-\mathrm{Thr}^{172}$ was increased. However, these effects were reversed following preincubation with Compound $\mathrm{C}$. The results suggest that pharmacological activation of AMPK could inhibit both cell proliferation and collagen secretion in cardiac fibroblasts infected by CVB3. Cardiac fibroblasts and collagen secretion are the key causes of myocardial fibrosis, which are characterized by excessive synthesis and accumulation of extracellular matrix proteins (28). In the later stage of CVB3-induced chronic myocarditis, cardiac fibrosis is an important pathogenic factor contributing to serious cardiovascular diseases by impairing ventricular contractility and functionality (3). The inhibitive effects of AMPK activation in CVB3-infected cardiac fibroblasts proliferation may be useful in the development of effective therapies for CVB3-induced myocarditis and dilated cardiomyopathy.

In conclusion, to the best of our knowledge, this is the first report of an inhibitive role of pharmacological AMPK activation in CVB3-induced cardiac fibroblast proliferation. This finding may be helpful for the future design of therapeutic approaches for treating cardiac fibrosis caused by chronic viral infection, such as CVB3-induced myocarditis.

\section{Acknowledgements}

This study was supported by the Natural Science Foundation of China (81200161) and Wuxi Hospital Management Center Project (YGZXM14012).

\section{References}

1. Massilamany C, Huber SA, Cunningham MW and Reddy J: Relevance of molecular mimicry in the mediation of infectious myocarditis. J Cardiovasc Transl Res 7: 165-171, 2014.

2. Antoniak S and Mackman N: Coagulation, protease-activated receptors, and viral myocarditis. J Cardiovasc Transl Res 7: 203-211, 2014.

3. Cao Y, Xu W and Xiong S: Adoptive transfer of regulatory T cells protects against Coxsackievirus B3-induced cardiac fibrosis. PLoS One 8: e74955, 2013.

4. Shen Y, Xu W, Chu YW, Wang Y, Liu QS and Xiong SD: Coxsackievirus group $\mathrm{B}$ type 3 infection upregulates expression of monocyte chemoattractant protein 1 in cardiac myocytes, which leads to enhanced migration of mononuclear cells in viral myocarditis. J Virol 78: 12548-12556, 2004.

5. Lindner D, Li J, Savvatis K, Klingel K, Blankenberg S, Tschöpe C and Westermann D: Cardiac fibroblasts aggravate viral myocarditis: Cell specific coxsackievirus B3 replication. Mediators Inflamm 2014: 519528, 2014.

6. Brown RD, Ambler SK, Mitchell MD and Long CS: The cardiac fibroblast: Therapeutic target in myocardial remodeling and failure. Annu Rev Pharmacol Toxicol 45: 657-687, 2005.

7. Hardie DG: AMPK: Positive and negative regulation and its role in whole-body energy homeostasis. Curr Opin Cell Biol 33: 1-7, 2015.

8. Stuck BJ, Lenski M, Böhm M and Laufs U: Metabolic switch and hypertrophy of cardiomyocytes following treatment with angiotensin II are prevented by AMP-activated protein kinase. J Biol Chem 283: 32562-32569, 2008.

9. Zhang CX, Pan SN, Meng RS, Peng CQ, Xiong ZJ, Chen BL, Chen GQ, Yao FJ, Chen YL, Ma YD and Dong YG: Metformin attenuates ventricular hypertrophy by activating the AMP-activated protein kinase-endothelial nitric oxide synthase pathway in rats. Clin Exp Pharmacol Physiol 38: 55-62, 2011.

10. Xie W, Wang L, Dai Q, Yu H, He X, Xiong J, Sheng H, Zhang D, Xin R, Qi Y, et al: Activation of AMPK restricts coxsackievirus B3 replication by inhibiting lipid accumulation. J Mol Cell Cardiol 85: 155-167, 2015.

11. Reed LJ and Muench H: A simple method of estimating fifty percent endpoints. Am J Hyg 27: 493-497, 1938.

12. Liu MY, Wu DL, Liu NH, Meng QW and Meng FC: $1 \mathrm{~A}$ and 3D gene sequences of coxsackievirus B3 strain CC: Variation and phylogenetic analysis. DNA Seq 19: 8-12, 2008.

13. Leipner C, Grün K, Schneider I, Glück B, Sigusch HH and Stelzner A: Coxsackievirus B3-induced myocarditis: Differences in the immune response of $\mathrm{C} 57 \mathrm{BL} / 6$ and $\mathrm{Balb} / \mathrm{c}$ mice. Med Microbiol Immunol 193: 141-147, 2004.

14. Chapman NM and Kim KS: Persistent coxsackievirus infection: Enterovirus persistence in chronic myocarditis and dilated cardiomyopathy. Curr Top Microbiol Immunol 323: 275-292, 2008. 
15. Pankuweit $\mathrm{S}$ and Klingel K: Viral myocarditis: From experimental models to molecular diagnosis in patients. Heart Fail Rev 18: 683-702, 2013.

16. Bhattacharjee A and Bansal M: Collagen structure: The Madras triple helix and the current scenario. IUBMB Life 57: 161-172, 2005.

17. Kasyanov V, Moreno-Rodriguez RA, Kalejs M, Ozolanta I, Stradins P, Wen X, Yao H and Mironov V: Age-related analysis of structural, biochemical and mechanical properties of the porcine mitral heart valve leaflets. Connect Tissue Res 54: 394-402, 2013

18. Liu WY and Jiang RS: Advances in the research of AMPK and its subunit genes. Pak J Biol Sci 16: 1459-1468, 2013.

19. Xiao B, Sanders MJ, Underwood E, Heath R, Mayer FV, Carmena D, Jing C, Walker PA, Eccleston JF, Haire LF, et al: Structure of mammalian AMPK and its regulation by ADP Nature 472: 230-233, 2011.

20. Baron SJ, Li J, Russell RR III, Neumann D, Miller EJ, Tuerk R, Wallimann T, Hurley RL, Witters LA and Young LH: Dual mechanisms regulating AMPK kinase action in the ischemic heart. Circ Res 96: 337-345, 2005.

21. Ma Y, Wang J, Gao J, Yang H, Wang Y, Manithody C, Li J and Rezaie AR: Antithrombin up-regulates AMP-activated protein kinase signalling during myocardial ischaemia/reperfusion injury. Thromb Haemost 113: 338-349, 2015.

22. Li Y, Chen C, Yao F, Su Q, Liu D, Xue R, Dai G, Fang R, Zeng J, Chen Y, et al: AMPK inhibits cardiac hypertrophy by promoting autophagy via mTORC1. Arch Biochem Biophys 558: 79-86, 2014.
23. Beauloye C, Bertrand L, Horman S and Hue L: AMPK activation, a preventive therapeutic target in the transition from cardiac injury to heart failure. Cardiovasc Res 90: 224-233, 2011.

24. Kim AS, Miller EJ, Wright TM, Li J, Qi D, Atsina K, Zaha V, Sakamoto K and Young LH: A small molecule AMPK activator protects the heart against ischemia-reperfusion injury. J Mol Cell Cardiol 51: 24-32, 2011

25. Paiva MA, Rutter-Locher Z, Gonçalves LM, Providência LA, Davidson SM, Yellon DM and Mocanu MM: Enhancing AMPK activation during ischemia protects the diabetic heart against reperfusion injury. Am J Physiol Heart Circ Physiol 300: H2123-H2134, 2011.

26. Li HL, Yin R, Chen D, Liu D, Wang D, Yang Q and Dong YG: Long-term activation of adenosine monophosphate-activated protein kinase attenuates pressure-overload-induced cardiac hypertrophy. J Cell Biochem 100: 1086-1099, 2007.

27. Gundewar S, Calvert JW, Jha S, Toedt-Pingel I, Ji SY, Nunez D, Ramachandran A, Anaya-Cisneros M, Tian R and Lefer DJ: Activation of AMP-activated protein kinase by metformin improves left ventricular function and survival in heart failure. Circ Res 104: 403-411, 2009.

28. Yamazaki KG, Gonzalez E and Zambon AC: Crosstalk between the renin-angiotensin system and the advance glycation end product axis in the heart: Role of the cardiac fibroblast. J Cardiovasc Transl Res 5: 805-813, 2012. 